\title{
Postponement of Death by Statin Use: a Systematic Review and Meta-analysis of Randomized Clinical Trials
}

\author{
Morten Rix Hansen, MD, PhD ${ }^{1,2,3}$, Asbjørn Hróbjartsson, MD, PhD ${ }^{4,5,6}$, \\ Anton Pottegård, MScPharm, $P h D^{7}$, Per Damkier, $M D, P h D^{2,7}$, \\ Kasper Søltoft Larsen, MD, $P h D^{7}$, Kenneth Grønkjaer Madsen, $M D^{7}$, \\ René dePont Christensen, M.Sc, PhD ${ }^{8}$, Malene Elisa Lopez Kristensen, MScPharm?', \\ Palle Mark Christensen, MD, PhD ${ }^{7}$, and Jesper Hallas, MD, DMSc ${ }^{1,2}$
}

${ }^{1}$ Clinical Pharmacology and Pharmacy, University of Southern Denmark, Odense, Denmark; ${ }^{2}$ Department of Clinical Biochemistry and Pharmacology, Odense University Hospital, Odense, Denmark; ${ }^{3}$ Department of Clinical Pharmacology, Aarhus University Hospital, Aarhus, Denmark; ${ }^{4}$ Center for Evidence-Based Medicine Odense, University of Southern Denmark, Sønderborg, Denmark; ${ }^{5}$ Department of Clinical Research, University of Southern Denmark, Sønderborg, Denmark; ${ }^{6}$ Odense Explorative Patient data Network (OPEN), Odense University Hospital, Odense, Denmark; ${ }^{7}$ Department of Clinical Research, University of Southern Denmark, Sønderborg, Denmark; ${ }^{8}$ Research Unit for General Practice, Odense, Denmark.

BACKGROUND: The average postponement of the outcome (gain in time to event) has been proposed as a measure to convey the effect of preventive medications. Among its advantages over number needed to treat and relative risk reduction is a better intuitive understanding among lay persons.

OBJECTIVES: To develop a novel approach for modeling outcome postponement achieved within a trial's duration, based on published trial data and to present a formalized meta-analysis of modeled outcome postponement for allcause mortality in statin trials.

METHODS: The outcome postponement was modeled on the basis of the hazard ratio or relative risk, the mortality rate in the placebo group and the trial's duration. Outcome postponement was subjected to a meta-analysis. We also estimated the average outcome postponement as the area between Kaplan-Meier curves. Statin trials were identified through a systematic review.

RESULTS: The median modeled outcome postponement was 10.0 days (interquartile range, 2.9-19.5 days). Metaanalysis of 16 trials provided a summary estimate of outcome postponement for all-cause mortality of 12.6 days, with a 95\% postponement interval (PI) of 7.1-18.0. For primary, secondary, and mixed prevention trials, respectively, outcome postponements were 10.2 days (PI, 4.016.3), 17.4 days (PI, 6.0-28.8), and 8.5 days (PI, 1.9-15.0). CONCLUSIONS: The modeled outcome postponement is amenable to meta-analysis and may be a useful approach for presenting the benefits of preventive interventions. Statin treatment results in a small increase of average survival within the duration of a trial.

SYSTEMATIC REVIEW REGISTRATION: The systematic review was registered in PROSPERO [CRD42016037507].

Electronic supplementary material The online version of this article (https://doi.org/10.1007/s11606-019-05024-4) contains supplementary material, which is available to authorized users.

Received February 26, 2018

Revised October 12, 2018

Accepted March 21, 2019

Published online May 9, 2019
J Gen Intern Med 34(8):1607-14

DOI: $10.1007 / \mathrm{s} 11606-019-05024-4$

(c) Society of General Internal Medicine 2019

\section{INTRODUCTION}

One challenge in the practice of medicine lies in adequately explaining the effects of a proposed intervention to enable a patient to make an informed decision. With regard to preventive interventions, such as statin use, effect size is traditionally expressed as relative/absolute risk reductions or "number needed to treat" (NNT). However, such measures are not necessarily best for conveying intervention effect. ${ }^{1,2}$

When contemplating preventative treatment, the additional time free of an undesirable clinical event can be considered more relevant. This average postponement of the study outcome represents an alternative to traditional effect measures for preventive treatment. ${ }^{3,4}$ It has been shown that patients are responsive to outcome postponement, i.e., their chance of accepting the treatment changes increases when they are presented with higher values of outcome postponement. ${ }^{5}$ On the other hand, even extreme differences in the presented values of NNT do not lead to greater or lower rates of treatment acceptance. ${ }^{6-8}$ Moreover, NNT conveys a "lottery-like" understanding of how the treatment effect is distributed, potentially suggesting that the risk of death is influenced in only 1 in 40 treated patients. As statin treatment reduces cholesterol levels in nearly all treated patients ${ }^{9}$ and a clear correlation between LDL cholesterol lowering and mortality has been demonstrated, ${ }^{10}$ it thus seems more plausible that statins slow atherosclerotic progression to some extent and thereby potentially delay death in all persons receiving treatment.

Methodologically, the average postponement accrued during a trial's duration can be estimated as the area between the survival curves for patients receiving the drug and placebo. ${ }^{4}$ Kristensen et al. ${ }^{5}$ recently published a systematic review of 11 
randomized clinical trials of statin use, each including at least 1000 patients. They estimated that median postponement of all-cause mortality within the trial duration was 3.2 days for primary prevention and 4.1 days for secondary prevention. ${ }^{4}$ However, their approach has two important weaknesses. First, a Kaplan-Meier survival curve for the outcome in question must be available. Second, measuring the area between survival curves does not allow for calculation of the variance of outcome postponement in the single trial, and thus outcome postponement cannot be subjected to meta-analysis using this approach.

In this study, we present a method for the meta-analysis of outcome postponement based on summary statistics from RCTs. We demonstrate the application of this method to estimate the average postponement of all-cause mortality based on meta-analysis of large placebo-controlled statin trials.

\section{METHODS}

\section{Data Sources and Searches}

We searched MEDLINE (search index date: May 1, 2015) using the following MeSH terms: statins, placebo, and random*. We also searched ClinicalTrials.gov (search index date: December 1, 2015) using the following terms: "statins" AND "placebo" (interventional). We further screened the reference lists of the included papers, but identified no additional studies for inclusion.

\section{Study Selection}

Our meta-analysis included randomized controlled trials of $\geq$ 1000 patients, in which a statin intervention (any type) was compared with placebo using a predefined primary or secondary outcome of death by any cause and having a minimum trial follow-up of 2 years. Furthermore, we only used the original publication, i.e., we excluded sub-studies of the original trial, and we excluded trials which investigate a pediatric population ( $<18$ years). Lastly, we excluded trials that did not provide parameters required for postponement estimation. Initially, we screened all abstracts for eligibility. We then extracted fulltexts articles, which we categorized with reasons for exclusion (Table e1-online).

\section{Data Extraction and Quality Assessment}

Two authors (MRH and KGM) independently extracted trial characteristics and outcome data from each included trial. Any discrepancies were resolved by consensus. The extracted trial characteristics included the trial's duration; whether it represented primary, secondary, or mixed intervention; and baseline LDL cholesterol level. The effect data included hazard ratio (HR) or relative risk (RR) and 95\% confidence interval (CI), and the mortality rate or cumulative mortality in the placebo group. We defined primary prevention trials as trials in which no patients had manifest cardiovascular disease at baseline, secondary prevention trials as those in which all of the patients had cardiovascular disease, and mixed prevention trials as trials including patients with and without cardiovascular disease. Two physicians independently performed trial classification.

The assessment of bias was performed using the Cochrane's risk of bias tool. ${ }^{11}$

If the paper did not report an estimated mortality rate in the placebo group, we used the following hierarchy of alternative measures: (a) the mortality rate among patients randomized to placebo as calculated by us based on the number of randomized patients, number of outcomes, and average follow-up; (b) the cumulative mortality among patients randomized to placebo as reported in the paper; (c) the cumulative mortality among patients randomized to placebo as determined from the Kaplan-Meier survival curve; and (d) the mortality rate among patients randomized to placebo, as calculated by us based on the number of randomized patients, number of events, and median follow-up. The latter approach may overestimate or underestimate the mortality rate, depending on whether the median follow-up is higher or lower than the average.

For trials that reported relative risk $(n=6)$ instead of hazard ratio as the outcome measure, we used relative risk as a proxy for hazard ratio. These measures are very similar when the cumulative risk values are low, e.g., below $20 \% .{ }^{11}$ None of the six trials that reported RR had a cumulative mortality risk of $>15 \%$.

\section{Data Analysis: Outcome Postponement}

We have calculated outcome postponement of death in each trial. Postponement is described by the area between survival curves. We have used two different methods, pixel counting and our novel mathematical model to estimate this area, which equals the postponement (gain in time) achieved in the active group. For details about the methods behind estimation of postponement from the mathematical model and pixel counting, see appendixes A- and B-online.

\section{Data Analysis: Meta-analysis}

We performed a meta-analysis of the postponement of all-cause death using inverse variance weighting and random effects models (STATA 14, Stata Corp., Texas) and using postponement intervals in place of confidence intervals. In order to describe effect modification by trial characteristics, we grouped trials according to (a) trial duration ( $>5$ years vs. $<5$ years); (b) primary vs. secondary intervention; (c) reporting by HR vs. RR as effect measures for all-cause mortality; (d) high vs. low overall risk of bias; (e) trials with the highest vs. lowest reduction in LDL cholesterol after 1 year and (f) whether or not the trial was terminated earlier than originally planned; (g) according to potency of the statin (low, medium, and high); and (h) the trials with the highest mortality rate vs. the trials with the lowest mortality rate. Moreover, we added a post hoc subgroup analysis comparing trials with high vs. low degree of patient 
cross-over (for example, from placebo to statin). We further investigated reporting bias using a funnel plot.

To adjust for heterogeneity attributable to the varying durations of the trials, we standardized the trial duration to 5 years in the modeled postponement. Essentially, this modeled the area between survival curves for 5 years of trial duration, based on the actually observed hazard ratio and cumulative mortality among untreated patients. Appendix C-online presents the equations used for standardization. Finally, to assist with interpretation of the postponement meta-analysis, we performed a HR-based meta-analysis of all-cause mortality.

The systematic review was registered in PROSPERO [CRD42016037507].

\section{RESULTS}

\section{Trial Retrieval}

We identified 115 potentially eligible trials, 16 of which met our inclusion criteria (Fig. 1, Table e1-online). Kaplan-Meier survival curves were available for eight of the trials, and all 16 trials presented the variables required to model the area between survival curves. Table 1 presents the trial characteristics (statin type, number of patients, trial duration, and LDL status). Two $(12.5 \%)$ were for primary prevention, six $(37.5 \%)$ for secondary prevention, and eight (50\%) for mixed prevention.

\section{Outcome Postponement}

Table 2 presents the estimated outcome postponement based on pixel counting and modeling. The median values were 10.6 days with the pixel counting method, and 8.9 days with the modeling method. We found strong agreement between these two methods, a slope of 0.95 with linear regression and a parametric Pearson correlation coefficient of $r=0.94$. Across all trials, the summary estimate of outcome postponement was 12.6 days (PI, 7.1-18.0). The $I^{2}$ value was 59.5, indicating moderate heterogeneity $(P=.001)$. After standardization to a trial duration of 5 years, the estimated summary outcome postponement for all trials was 12.8 days (PI, 8.9-16.7), and $I^{2}$ decreased to $22.3 \%$ which was no longer statistically significant $(P=.179)$ (Fig. 2).

\section{Subgroups}

Table 3 shows the results of subgroup analyses by trial characteristics. Briefly, the modeled outcome postponements were 10.2 days (PI, 4.1-16.3) for primary prevention, 17.4 days (PI, 6.0-28.8) for secondary prevention, and 8.5 days (PI, 1.915.1) for mixed prevention. HR-based meta-analyses produced a summary HR of 0.89 (CI, 0.84-0.94) for all trials, 0.78 (CI, 0.67-0.92) for primary prevention trials, 0.85 (CI, 0.75-0.96) for secondary prevention trials, and 0.92 (CI, 0.880.97) for mixed prevention trials (Fig. 3).

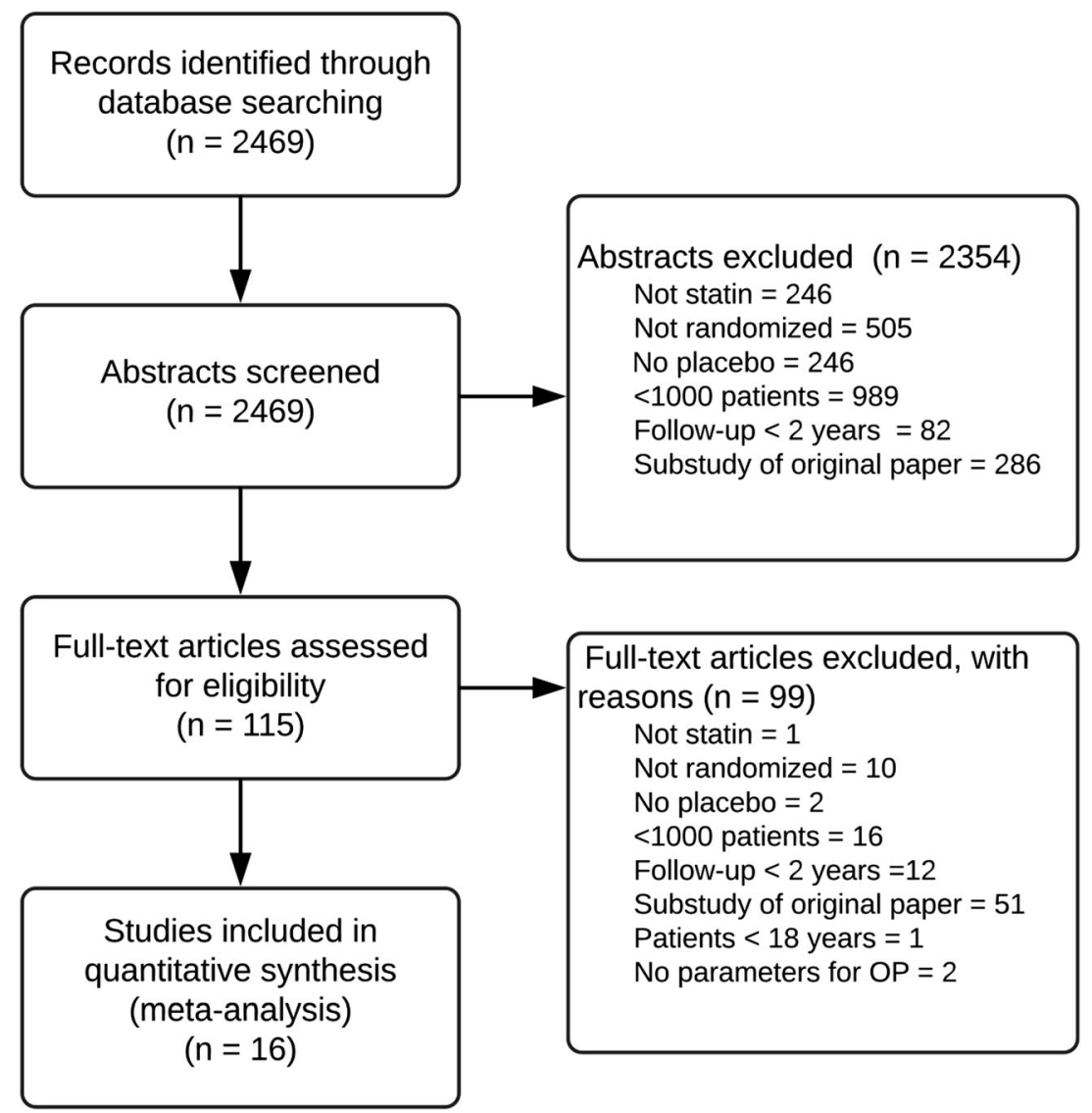

Figure 1 Flowchart of search to identify randomized, placebo-controlled statin trials. 

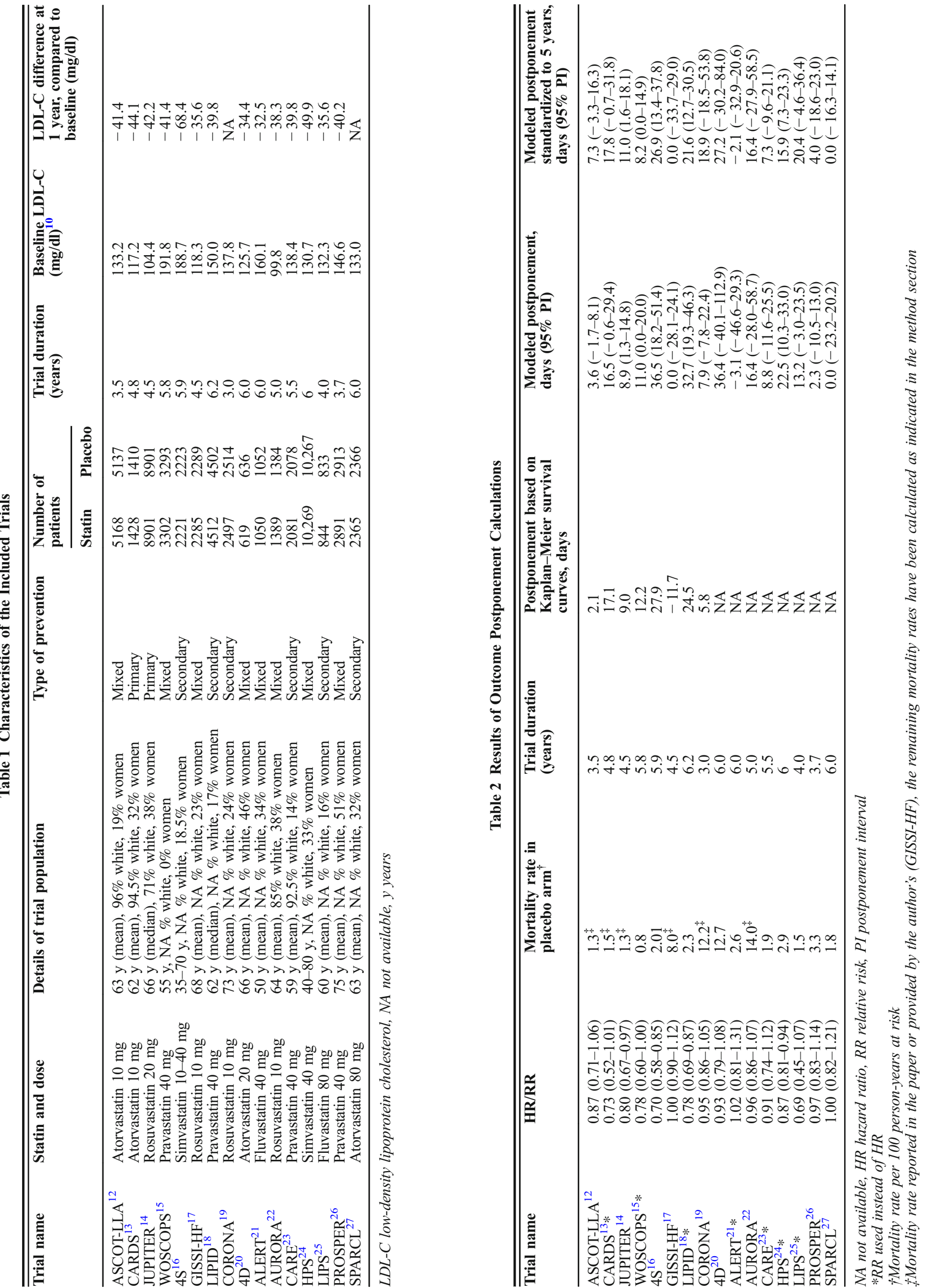


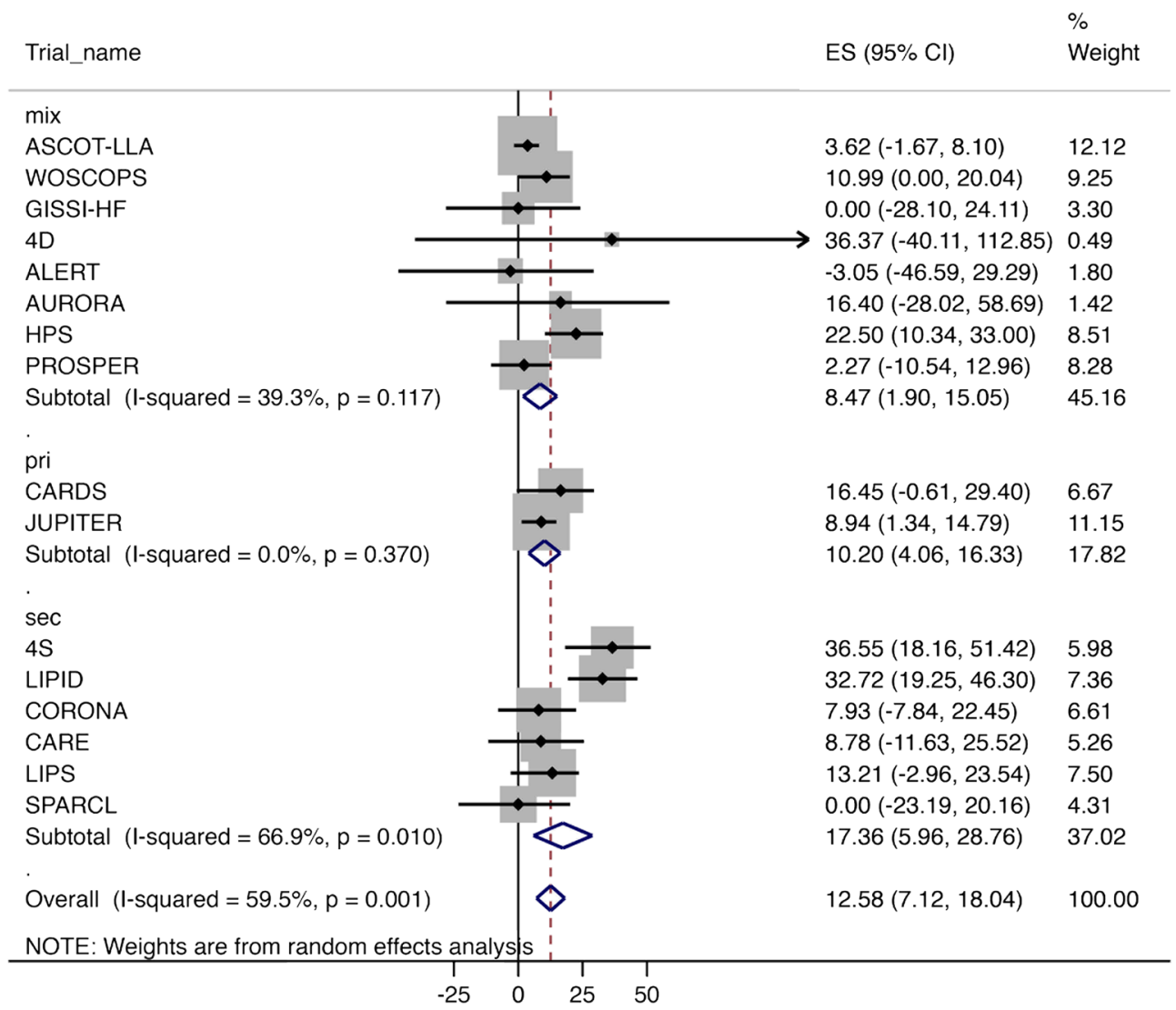

Figure 2 Forest plots of postponement of all-cause mortality.

Table 3 Subgroup Meta-analyses of Postponement of All-Cause Mortality According to Trial Characteristics

\begin{tabular}{|c|c|c|c|c|c|c|c|}
\hline Subgroup & $\begin{array}{l}\text { Number } \\
\text { of trials }\end{array}$ & $\begin{array}{l}\text { Outcome } \\
\text { postponement, } \\
\text { days }(95 \% \mathrm{PI})\end{array}$ & $I^{2}$ & $\begin{array}{l}\text { Outcome postponement } \\
\text { standardized to } 5 \text { years, } \\
\text { days }(95 \% \text { PI) }\end{array}$ & $I^{2}$ & $\begin{array}{l}\text { HR-based } \\
\text { meta-analysis, } \\
\text { HR }(95 \% \text { CI })\end{array}$ & $I^{2}$ \\
\hline All trials & 16 & $12.6(7.1-18.0)$ & 60 & $12.8(8.8-16.7)$ & 22 & $0.89(0.84-0.94)$ & 47 \\
\hline Primary prevention & 2 & $10.2(4.1-16.3)$ & 0 & $12.4(5.0-19.8)$ & 0 & $0.78(0.67-0.92)$ & 0 \\
\hline Secondary prevention & 6 & $17.4(6.0-28.8)$ & 67 & $16.2(7.4-25.1)$ & 50 & $0.85(0.75-0.96)$ & 66 \\
\hline Mixed prevention & 8 & $8.5(1.9-15.0)$ & 39 & $10.0(5.5-14.5)$ & 0 & $0.92(0.88-0.97)$ & 12 \\
\hline Trial duration $<5$ years & 7 & $6.3(2.9-9.7)$ & 0 & $10.7(5.4-16.0)$ & 0 & $0.91(0.84-0.99)$ & 33 \\
\hline Trial duration $>5$ years & 9 & $18.6(9.5-27.7)$ & 52 & $13.6(7.3-19.9)$ & 47 & $0.88(0.81-0.94)$ & 52 \\
\hline Early trial termination & 3 & $18.6(4.0-33.3)$ & 79 & $16.4(9.1-23.6)$ & 33 & $0.78(0.71-0.86)$ & 0 \\
\hline Planned trial termination & 13 & $10.8(4.7-16.8)$ & 51 & $11.3(6.6-15.9)$ & 17 & $0.91(0.87-0.96)$ & 35 \\
\hline $\begin{array}{l}\text { High degree of switching } \\
\text { between groups* }\end{array}$ & 10 & $11.6(5.1-18.1)$ & 64 & $12.7(8.4-16.9)$ & 13 & $0.88(0.84-0.93)$ & 27 \\
\hline Unclear degree of switching between groups & 5 & $10.4(3.0-17.8)$ & 0 & $8.7(2.1-15.2)$ & 0 & $0.95(0.86-1.04)$ & 28 \\
\hline $\begin{array}{l}\text { Low degree of switching } \\
\text { between groups }\end{array}$ & 1 & $36.5(19.9-53.2)$ & - & $27.0(14.7-39.2)$ & - & $0.70(0.58-0.85)$ & - \\
\hline $\begin{array}{l}\text { Trials with the least LDL } \\
\text { reduction at } 1 \text { year }\end{array}$ & 5 & $10.3(-0.5-21.1)$ & 0 & $11.1(-2.2-24.5)$ & 0 & $0.97(0.90-1.03)$ & 0 \\
\hline Trials with the greatest LDL reduction at 1 year & 9 & $14.7(7.5-21.9)$ & 77 & $13.7(9.1-18.4)$ & 41 & $0.83(0.78-0.89)$ & 28 \\
\hline High potency statins & 6 & $8.1(2.3-13.7)$ & 0 & $8.92(2.1-15.8)$ & 0 & $0.95(0.90-1.00)$ & 0 \\
\hline Medium potency statins & 5 & $17.3(5.6-29.0)$ & 82 & $16.6(9.8-23.4)$ & 37 & $0.81(0.73-0.90)$ & 33 \\
\hline Low potency statins & 5 & $12.35(0.4-24.3)$ & 68 & $10.8(2.8-18.9)$ & 46 & $0.88(0.78-0.99)$ & 47 \\
\hline $\begin{array}{l}\text { Trials with the highest } \\
\text { mortality rate }\end{array}$ & 8 & $13.9(3.3-24.5)$ & 56 & $16.5(10.8-21.6)$ & 0 & $0.92(0.87-0.98)$ & 24 \\
\hline $\begin{array}{l}\text { Trials with the lowest } \\
\text { mortality rate }\end{array}$ & 8 & $11.2(5.1-17.4)$ & 59 & $11.6(6.2-16.9)$ & 38 & $0.82(0.75-0.90)$ & 50 \\
\hline
\end{tabular}

HR hazard ratio, PI postponement interval

*High incidence of switching was defined as above $4 \%$

†Theshold for highest reduction was defined as $\leq 39.8 \mathrm{mg} / \mathrm{dl}$. Two trials did not report LDL reduction

$\$$ Threshold for lowest mortality rate was defined as $\leq 0.022$ per 100 person-years at risk (median) 


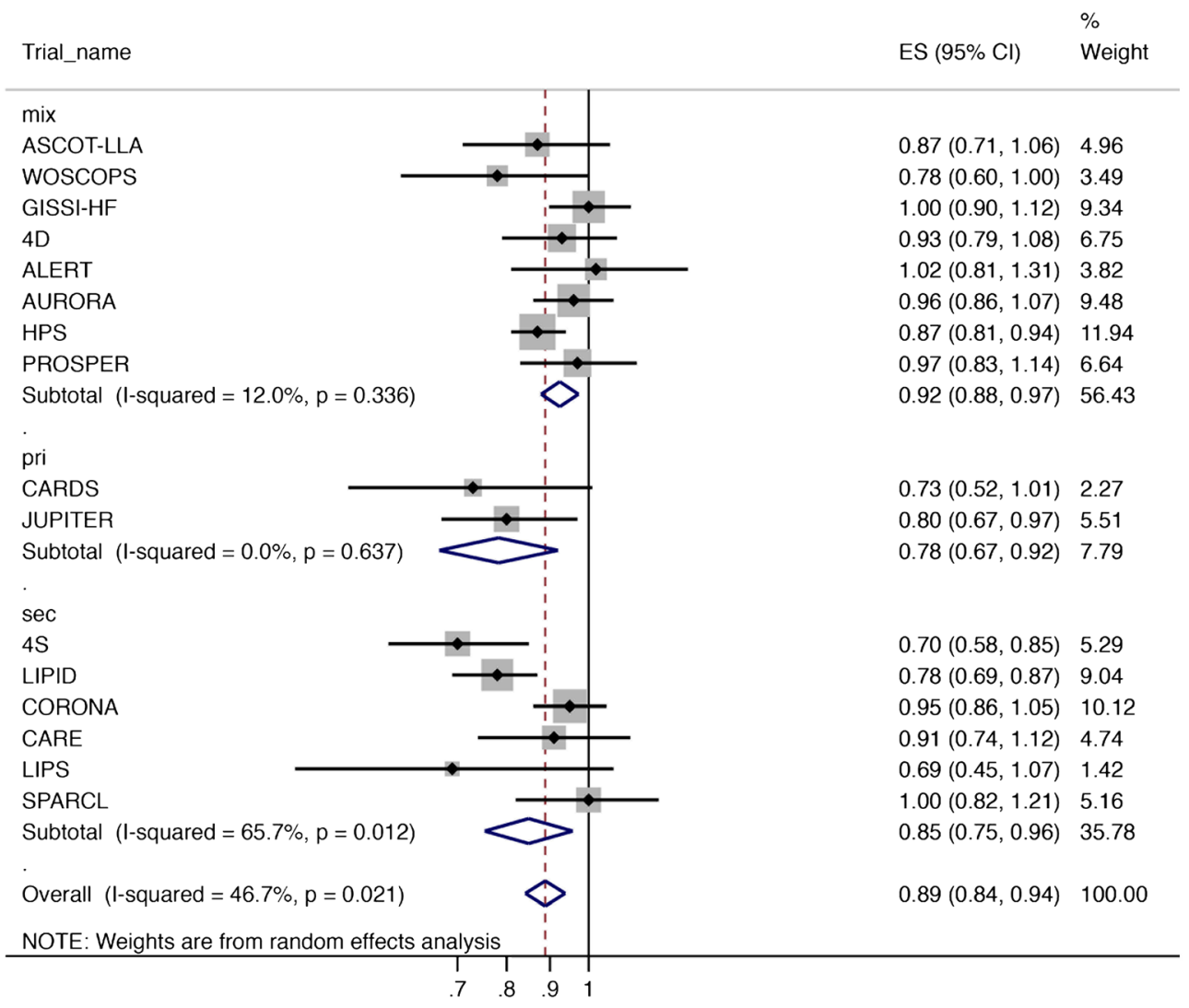

Figure 3 Forest plot of hazard ratios in the trials.

\section{Risk of Bias}

All included trials were large and had published or accessible protocols, concealed allocation, and blinding. All trials had a low overall risk of bias as determined using the Cochrane risk of bias tool. ${ }^{11}$ Treatment switches were a possible concern, as some trials reported that considerable proportions of patients (range, 4.8-25.4\%) assigned to the placebo group switched to statin treatment or were assigned to the statin group but stopped their treatment. Outcome postponements were 11.6 days (PI, 5.1-18.1) in the ten trials with high cumulative incidence ( $>4 \%)$ of treatment switches, 36.5 days (PI, 19.953.2) in the single trial with a low degree of switching, and 10.4 days (PI, 3.0-17.8) in the five trials with an unclear degree of switching.

\section{DISCUSSION}

Here, we investigated the effects of statin treatment on postponement of death and performed a meta-analysis. We found that statin treatment resulted in a small average increase of survival within the trials' duration. Meta-analysis of 16 large RCTs revealed a survival gain of 12.6 days (PI, 7.1-18.0) within the trial duration. We stratified on prevention type and demonstrated the largest postponement among the trials with secondary prevention, 17 days compared to 10 and 9 days in the primary and mixed prevention groups. We expected this result, as the largest relative risk reduction has previously been found in this group. ${ }^{10}$ We examined the effect of trial duration on postponement and found a much larger postponement among the trial's with a trial duration of 5 years and above, compared to below 5 years (19 days vs. 6 days). The difference nearly disappeared after standardization (14 days vs. 11 days), demonstrating the strong dependency of outcome postponement on trial duration. The proposed model has several important strengths. The model-derived area between survival curves showed strong agreement with the area between survival curves as measured by pixel counting. Additionally, the model does not require a Kaplan-Meier survival curve to determine the outcome postponement, increasing the applicability of this method to a wider range of trials. Lastly, the use of a confidence interval proxy for outcome postponement enabled meta-analysis.

The postponement of death from other interventions varies greatly. One recent review on medical interventions for cancer, approved by the EMA from 2009 to 2013, showed that only $51 \%$ of the drugs postponed death at all. The median followup was 5.4 years. ${ }^{28}$ A different study evaluated the effects of regular exercise and a calorie restricted diet in healthy males, demonstrating a postponement of death by 6.2 months. Lastly, smoking cessation in a high-risk population postponed death by around 31 months. ${ }^{4}$

As a tool for conveying treatment effects to patients, the concept of outcome postponement has important advantages 
over the number needed to treat (NNT). Most importantly, outcome postponement achieves greater responsiveness from patients. Surveys demonstrate that patients have the same likelihood of accepting a hypothetical treatment across NNT values ranging from 10 to $400 .{ }^{6,29}$ On the other hand, patients presented with benefits conveyed in terms of outcome postponement clearly discriminate between efficient and lessefficient treatments. Additionally, an NNT value may be criticized for conveying a "lottery-like" understanding of how treatment effect is distributed - for example, an NNT value of 40 could be wrongfully interpreted as meaning that 1 in 40 patients will experience all of the benefits treatment, while the remaining 39 experience no effect.

The most important clinical limitation of our model is that it does not capture the outcome postponement accrued after trial termination. Estimation of such benefits is difficult and heavily relies on untestable assumptions. For example, Marshall used extrapolations from the LIPID trial to estimate the gain in life expectancy from lifelong statin treatment (i.e., for the rest of their lives) and arrived at a gain of 7.9 years. However, Chang et al. used the same data in a different model and estimated a gain of 3.0 years. ${ }^{30}$ Using a model that incorporated mortality in different risk strata, Støvring et al. ${ }^{31}$ estimated that lifelong statin treatment was associated with survival gains ranging from 3 to 11 months. Franco et al. ${ }^{32}$ used lifetable techniques and reported outcome postponements of 2.0 and 2.4 years for lifelong statin use starting from 40 and 60 years of age, respectively. Some of these models apply the strong assumption that HR remains constant throughout the subject's lifetime, and most assume that all subjects can maintain lifelong statin treatment, which has been established as unrealistic in nearly all drug utilization studies. ${ }^{33,}{ }^{34}$ Using our presently described method, we modeled the mortality outcome postponement in a recent study that provides Kaplan-Meier curves for 20 years of follow-up of the WOSCOPS trial, estimating an outcome postponement of 152 days (PI, 70-236 days). ${ }^{35}$ Given the uncertainties of estimating survival gain after trial termination, it is important to emphasize that our calculated outcome postponement is that achieved within the trial's duration and should be considered an underestimate of the full outcome postponement including all post-trial follow-up. There is also a possibility of error by assuming that the survival curves would conform to an exponential decay function within the trial's running time or that this assumption is violated by the standardization to 5 years. However, the fact that there is good agreement between modeled postponement and postponement measured by pixel counting suggests that such violations are insignificant.

Other models have been proposed for estimating and presenting outcome postponement during a trial's duration. Lytsy $^{36}$ described a "delay of events" model that essentially estimates the average outcome postponement among patients who experienced the outcome, rather than among all patients randomized to receive treatment as in our model. The estimated outcome postponement in the $4 \mathrm{~S}$ study ${ }^{16}$ which they used as motivating case was 1.0 year, which was larger than our estimate of 36.5 days. Notably, the "delay of event" measure seems to have little clinical utility, as it only applies to patients who die during the course of the trial, e.g., a population that cannot be identified at baseline. Additionally, Royston and Parmar ${ }^{37,38}$ developed the concept of restricted mean survival time (RMST), which generalizes outcome postponement. The RMST approach is fully developed from a theoretical and practical point of view, and it is the method of choice if data are available at the individual level.

From a clinical viewpoint, it would be a cautious and pragmatic approach to offer statins in accordance with the prevailing guidelines, i.e., as secondary prevention for all patients with manifest atherosclerotic disease and as primary prevention for certain high-risk patients. Statins are inexpensive, usually well tolerated, ${ }^{39}$ and show a favorable costutility. ${ }^{40}$ Based on the present evidence that statins do not have a large effect on postponement of all-cause mortality within a trial's duration, physicians and patients may be more inclined to discontinue treatment in patients showing intolerance to statins or having a short life expectancy.

In summary, we have developed a simple method for estimating outcome postponement based on summary measures that are almost universally available. This method is amenable to meta-analyses, and we believe that it may be a useful approach to presenting the benefits of preventive interventions to patients. We envisioned outcome postponement used as complementary to the prevailing measures. With regard to the subject matter in our present meta-analysis, statin treatment resulted in a small gain in average survival within the trials' duration. However, statins reduce the risk of cardiovascular outcomes, which could add value to the drug, from the patient's perspective, irrespective of the effect on all-cause mortality.

Acknowledgments: We thank Jesper Urban Pedersen, Senior Creative, for the technical advice regarding the use of Adobe Photoshop and Manan Pareek, M.D., for reviewing the statin trial classifications.

Corresponding Author: Morten Rix Hansen, MD, PhD; Clinical Pharmacology and Pharmacy, University of Southern Denmark, J.B. Winsløwsvej 19, 2 5000, Odense, Denmark (e-mail: mrix@health.sdu. $d k)$.

Authors' Contribution All authors have made substantial contributions to conception and design, acquisition of data, or analysis and interpretation of data. They have all participated in drafting of the paper or revising it critically for important intellectual content. All authors have read and approved the final version of the manuscript.

Funding This study was funded by the University of Southern Denmark. The funder played no role in the design, conduct, or reporting.

\section{Compliance with Ethical Standards:}

Conflict of Interest: All authors have completed the ICMJE uniform disclosure form at www.icmje.org/coi disclosure.pdf: The authors declare that they do not have a conflict of interest. 


\section{REFERENCES}

1. Hux JE, Naylor CD. Communicating the benefits of chronic preventive therapy does the format of efficacy data determine patients' acceptance of treatment? Med Decis Mak 1995;15(2):152-157.

2. Malenka DJ, Baron JA, Johansen S, Wahrenberger JW, Ross JM. The framing effect of relative and absolute risk. J Gen Intern Med 1993;8(10):543-548.

3. Laupacis A, Sackett DL, Roberts RS. An assessment of clinically useful measures of the consequences of treatment. N Engl J Med 1988;318(26):17281733. https://doi.org/10.1056/NEJM198806303182605

4. Wright JC, Weinstein MC. Gains in life expectancy from medical interventions-standardizing data on outcomes. N Engl J Med 1998;339(6):380-386.

5. Kristensen ML, Christensen PM, Hallas J. The effect of statins on average survival in randomised trials, an analysis of end point postponement. BMJ Open 2015;5(9):e007118. https://doi.org/10.1136/ bmjopen-2014-007118

6. Christensen PM, Brosen $\mathbf{K}$, Brixen $\mathbf{K}$, Andersen $\mathbf{M}$, Kristiansen IS. A randomized trial of laypersons' perception of the benefit of osteoporosis therapy: number needed to treat versus postponement of hip fracture. Clin Ther 2003;25(10):2575-2585.

7. Morris J, Hammitt JK. Using life expectancy to communicate benefits of health care programs in contingent valuation studies. Med Decis Mak 2001;21(6):468-478.

8. Halvorsen PA, Aasland OG, Kristiansen IS. Decisions on statin therapy by patients' opinions about survival gains: cross sectional survey of general practitioners. BMC Fam Pract 2015;16(1). https://doi.org/10. 1186/s12875-015-0288-8

9. Kapur NK, Musunuru $\mathbf{K}$, et al. Clinical efficacy and safety of statins in managing cardiovascular risk. Vasc Health Risk Manag 2008;4(2):341.

10. Trialists CT, et al. Efficacy and safety of more intensive lowering of LDL cholesterol: a meta-analysis of data from 170000 participants in 26 randomised trials. Lancet 2010;376(9753):1670-1681.

11. Higgins JPT, Green S. Cochrane Handbook for Systematic Reviews of Interventions. Vol 2008.

12. Sever PS, Dahlof B, Poulter NR, et al. Prevention of coronary and stroke events with atorvastatin in hypertensive patients who have average or lowerthan-average cholesterol concentrations, in the Anglo-Scandinavian Cardiac Outcomes Trial-Lipid Lowering Arm (ASCOT-LLA): a multicentre randomised controlled trial. Lancet 2003;361(9364):1149-1158. https://doi.org/10. 1016/S0140-6736(03) 12948-0

13. Colhoun HM, Betteridge DJ, Durrington PN, et al. Primary prevention of cardiovascular disease with atorvastatin in type 2 diabetes in the Collaborative Atorvastatin Diabetes Study (CARDS): multicentre randomised placebo-controlled trial. Lancet 2004;364(9435):685-696. doi:https://doi.org/10.1016/S0140-6736(04) 16895-5

14. Ridker PM, Danielson E, Fonseca FA, et al. Rosuvastatin to prevent vascular events in men and women with elevated C-reactive protein. N Engl J Med 2008;359(21):2195.

15. Shepherd J, Cobbe SM, Ford I, et al. Prevention of coronary heart disease with pravastatin in men with hypercholesterolemia. N Engl J Med 1995;333(20): 1301-1308.

16. Randomised trial of cholesterol lowering in 4444 patients with coronary heart disease: the Scandinavian Simvastatin Survival Study (4S). Lancet. 1994;344(8934): 1383-1389

17. Tavazzi L, Maggioni AP, Marchioli R, et al. Effect of rosuvastatin in patients with chronic heart failure (the GISSI-HF trial): a randomised, double-blind, placebo-controlled trial. Lancet 2008;372(9645):12311239. doi:https://doi.org/10.1016/S0140-6736(08)61240-4

18. Prevention of cardiovascular events and death with pravastatin in patients with coronary heart disease and a broad range of initial cholesterol levels. The Long-Term Intervention with Pravastatin in Ischaemic Disease (LIPID) Study Group. N Engl J Med 1998;339(19):1349-1357. doi:https://doi.org/10. 1056/NEJM199811053391902

19. Kjekshus J, Apetrei E, Barrios V, et al. Rosuvastatin in older patients with systolic heart failure. N Engl J Med 2007;357(22):2248-2261.

20. Wanner C, Krane V, Marz W, et al. Atorvastatin in patients with type 2 diabetes mellitus undergoing hemodialysis. N Engl J Med 2005;353(3):238-248. doi:https://doi.org/10.1056/NEJMoa043545

21. Holdaas H, Fellström B, Jardine AG, et al. Effect of fluvastatin on cardiac outcomes in renal transplant recipients: a multicentre, randomised, placebo-controlled trial. Lancet 2003;361(9374):2024-2031.
22. Fellstrom BC, Jardine AG, Schmieder RE, et al. Rosuvastatin and cardiovascular events in patients undergoing hemodialysis. N Engl J Med 2009;360(14):1395-1407. doi:https://doi.org/10.1056/ NEJMoa0810177

23. Sacks FM, Pfeffer MA, Moye LA, et al. The effect of pravastatin on coronary events after myocardial infarction in patients with average cholesterol levels. Cholesterol and Recurrent Events Trial investigators. N Engl J Med 1996;335(14):1001-1009. doi:https://doi.org/10.1056/ NEJM199610033351401

24. Heart Protection Study Collaborative Group. MRC/BHF Heart Protection Study of cholesterol lowering with simvastatin in 20,536 high-risk individuals: a randomised placebo-controlled trial. Lancet 2002;360(9326):7-22. doi:https://doi.org/10.1016/S0140-6736(02) 09327-3

25. Serruys PWJC, de Feyter P, Macaya C, et al. Fluvastatin for prevention of cardiac events following successful first percutaneous coronary intervention: a randomized controlled trial. JAMA J Am Med Assoc 2002;287(24):3215-3222.

26. Shepherd J, Blauw GJ, Murphy MB, et al. Pravastatin in elderly individuals at risk of vascular disease (PROSPER): a randomised controlled trial. Lancet. 2002;360(9346):1623-1630.

27. Amarenco P, Bogousslavsky J, Callahan A 3rd, et al. High-dose atorvastatin after stroke or transient ischemic attack. N Engl J Med 2006;355(6):549-559. doi:https://doi.org/10.1056/NEJMoa061894

28. Davis C, Naci H, Gurpinar E, Poplavska E, Pinto A, Aggarwal A. Availability of evidence of benefits on overall survival and quality of life of cancer drugs approved by European Medicines Agency: retrospective cohort study of drug approvals 2009-13. BMJ. 2017:j4530. doi:https:// doi.org/10.1136/bmj.j4530

29. Halvorsen PA, Selmer R, Kristiansen IS. Different ways to describe the benefits of risk-reducing treatments: a randomized trial. Ann Intern Med 2007; 146(12):848-856

30. Kristensen ML, Christensen PM, Hallas J. The effect of statins on average survival in randomised trials, an analysis of end point postponement, Web comments. BMJ Open 2015;5(9):e007118.

31. Stovring H, Harmsen CG, Wisloff T, et al. A competing risk approach for the European Heart SCORE model based on cause-specific and all-cause mortality. Eur J Prev Cardiol 2013;20(5):827-836. doi:https://doi.org/ $10.1177 / 2047487312445425$

32. Franco $\mathbf{O H}$. Effectiveness calculation in economic analysis: the case of statins for cardiovascular disease prevention. J Epidemiol Community Health 2006;60(10):839-845. doi:https://doi.org/10.1136/jech.2005.041251

33. Shah ND, Dunlay SM, Ting HH, et al. Long-term medication adherence after myocardial infarction: experience of a community. Am $\mathrm{J}$ Med 2009;122(10):961.e7-961.e13. doi:https://doi.org/10.1016/j.amjmed. 2008.12.021

34. Chan DC, Shrank WH, Cutler D, et al. Patient, physician, and payment predictors of statin adherence. Med Care 2010;48(3):196-202.

35. Ford I, Murray H, McCowan C, Packard CJ. Long-term safety and efficacy of lowering low-density lipoprotein cholesterol with statin therapy 20-year follow-up of West of Scotland Coronary Prevention Study. Circulation. 2016;133(11):1073-1080.

36. Lytsy P, Berglund L, Sundström J. A proposal for an additional clinical trial outcome measure assessing preventive effect as delay of events. Eur J Epidemiol 2012;27(12):903-909. doi:https://doi.org/10.1007/s10654012-9752-0

37. Dehbi H-M, Royston P, Hackshaw A. Life expectancy difference and life expectancy ratio: two measures of treatment effects in randomised trials with non-proportional hazards. BMJ 2017:j2250. doi:https://doi.org/10. 1136/bmj.j2250

38. Royston P, Parmar MK. Restricted mean survival time: an alternative to the hazard ratio for the design and analysis of randomized trials with a time-to-event outcome. BMC Med Res Methodol 2013;13(1):152.

39. Armitage $\mathbf{J}$. The safety of statins in clinical practice. Lancet 2007;370(9601): 1781-1790

40. Johannesson M, Jönsson B, Kjekshus J, Olsson AG, Pedersen TR. Wedel H. Cost effectiveness of simvastatin treatment to lower cholesterol levels in patients with coronary heart disease. N Engl J Med 1997;336(5):332-336.

Publisher's Note Springer Nature remains neutral with regard to jurisdictional claims in published maps and institutional affiliations. 